\title{
„Neurodermitis wird oft als Kinderkrankheit angesehen"
}

\author{
Über die wichtigsten Neuerungen der Leitlinie, das Problem eigenmächtiger \\ Eliminationsdiäten, die spezifische Immuntherapie als Therapieoption sowie \\ Lücken in der Versorgung gibt PD Dr. Marc Alexander Radtke Auskunft.
}

? Was sind die wichtigsten Neuerungen in der aktualisierten Leitlinie Neurodermitis?

PD Dr. med. Marc Alexander Radtke: Das Grundgerüst und die Kernaussagen sind erhalten geblieben. Es bleibt auch bei der Stufigkeit der Therapie, die sich nach dem Schweregrad richtet. Änderungen betreffen an verschiedenen Stellen hinzugekommene Evidenz. In der topischen Therapie ist das die proaktive Therapie sowie die rechtzeitige Einbeziehung von Immunmodulatoren und in der systemischen Therapie der hohe Stellenwert von Ciclosporin A, der in der Versorgung bisher zu wenig beachtet wird. Ciclosporin war in einer Studie der systemischen Kortisontherapie hochüberlegen. Es sollte daher bei schweren Formen und Versagen der topischen Therapie häufiger eingesetzt werden.

? Was ist bei der "proaktiven Nachbehandlung" $z$ u beachten?

Radtke: Durch die längerfristige intermittierende Anwendung von Glukokortikoiden oder Immunmodulatoren wird das Auftreten von Flares weniger wahrscheinlich. Die Neurodermitis ist eine sehr dynamische Erkrankung, bei der kutane Entzündungen und Juckreiz innerhalb von Stun-

\section{$y=-1$ \\ "Viele Patienten suchen keine dermatologische Hilfe, weil sie den Juckreiz inzwischen als gegebenen Zustand akzeptieren."}

PD Dr. med. Marc Alexander Radtke Institut für Versorgungsforschung in der Dermatologie, Universitätsklinikum Hamburg-Eppendorf den oder Minuten entstehen können. Indem man die - unerkannte - Anbahnung von Symptomen hemmt, erreicht man eine bessere Kontrolle über den Hautzustand. Natürlich muss man mit Augenmaß und abhängig vom individuellen Schweregrad und von der Lokalisation entscheiden, wie häufig und wie lange man eine solche Therapie durchführt. Wenn wiederholte Behandlungsphasen notwendig sind, wird man statt der topischen Glukokortikosteroide eher topische Immunmodulatoren verwenden, weil man die auch längerfristig einsetzen kann.

? Erstmals wird die spezifische Immuntherapie als Option „allein wegen der Neurodermitis" genannt. Ist davon eine Besserung der Neurodermitis zu erwarten?

Radtke: Das setzt natürlich voraus, dass eine Typ-1-Allergie, zum Beispiel gegen Pollen oder Tierhaare, als auslösendes und tragendes Element identifiziert ist. Dazu reicht nicht der Nachweis einer Sensibilisierung im RAST oder Pricktest, die Sensibilisierung muss sich auch durch eine Provokation oder eine eindeutige Korrelation als klinisch relevant erwiesen haben. Dann kann mit der spezifischen Immuntherapie in sehr vielen Fällen auch eine Verbesserung des Hautbefundes erreicht werden.

? In der Leitlinie wird betont, dass eine Eliminationsdiät nur bei nachgewiesener Allergie eingehalten werden sollte. Wird diese Bedingung zu wenig beachtet?

Radtke: Im Alltag wird generell zu wenig auf Nahrungsmittelallergien geachtet. Oft besteht eine Diskrepanz zwischen der subjektiven Wahrnehmung des Patienten hinsichtlich eines als auslösend empfundenen Nahrungsmittels und einer tatsächlichen Nahrungsmittelallergie. Wir empfehlen Neurodermitispatienten immer, ein Ernäh- rungstagebuch zu führen. Wenn im RAST eine Sensibilisierung gegen ein Nahrungsmittelallergen festgestellt wird, heißt das noch lange nicht, dass das Weglassen der entsprechenden Lebensmittel einen Nutzen hat. Mitunter werden Diäten durchgeführt, ohne dass eine klinische Korrelation besteht. Das ist in Phasen von Wachstum und Entwicklung besonders kritisch. Grundsätzlich sollte eine Diagnostik von Fachärzten durchgeführt worden sein, bevor auf eigenen Verdacht Lebensmittel weggelassen werden. Bei Eliminationsdiäten sollten immer Ernährungsberater hinzugezogen werden, damit eine gegebenenfalls notwendige Substitution stattfindet.

? Wie steht es sonst mit der Umsetzung der Leitlinien: Sind Patienten mit Neurodermitis in Deutschland gut versorgt? Radtke: Wir beobachten eine Unterschätzung der Altersprävalenz. Die Neurodermitis wird oft als Kinder- und Jugenderkrankung angesehen, die im Erwachsenenalter sistiert. Tatsächlich leiden viele Erwachsene weiterhin an der Erkrankung. Viele von ihnen suchen keine dermatologische Hilfe, weil sie den Juckreiz inzwischen als gegebenen Zustand akzeptieren. Es gibt daher eine hohe Dunkelziffer von erwachsenen Neurodermitispatienten. Ein Problem ist auch, dass wir bei der Neurodermitis lange nicht so viele Therapieoptionen haben wie etwa bei der Schuppenflechte. Für die systemische Therapie gibt es nur Ciclosporin, aber noch kein einziges Biologikum. Fast alle Systemtherapien unterliegen dem Off-label-Use. Dies ist für viele niedergelassene Ärzte immer noch eine bedeutende Hürde für den Einsatz.

? Ist in nächster Zeit mit neuen Therapieoptionen zu rechnen?

Radtke: Wir erwarten jetzt die Publikation der Phase-3-Studie mit Dupilumab, an der wir auch beteiligt waren. Die Ergebnisse sind vielversprechend. In der Biologikatherapie wäre das der erste große Meilenstein bei Neurodermitis. Auch in der topischen Therapie der Neurodermitis sind neue Wirkstoffe in der klinischen Erprobung.

Das Interview führte Dr. Beate Schumacher. 